\title{
Should women be offered cholesterol lowering drugs to prevent cardiovascular disease?
}

Scott M Grundy professor, Center for Human Nutrition, Departments of Clinical Nutrition and Internal Medicine, University of Texas Southwestern Medical Center at Dallas, 5323 Harry Hines Boulevard, Dallas, Texas 75390-9052, USA scott.grundy@utsouthwestern.edu

YES Primary and secondary trials show that cholesterol lowering drugs reduce the risk of cardiovascular disease events in men. ${ }^{12}$ Secondary prevention trials in women with established cardiovascular disease also show a reduction in risk. ${ }^{3-5}$ Meta-analyses also show that when all women with or without cardiovascular disease are combined, cholesterol lowering treatment produces benefit. ${ }^{67}$

Some investigators nevertheless argue that women without cardiovascular disease should not be given cholesterol lowering drugs because of insufficient evidence from primary prevention trials in women. ${ }^{8}$ They speculate that women with and without cardiovascular disease respond differently to the drugs; if true, women may not respond to treatment even when they have the same level of risk as men who do benefit. ${ }^{12}$ This speculation discounts prospective studies showing that raised cholesterol concentration is a major risk factor for first heart attack in both men and women. ${ }^{9}$

The pathological basis for a difference in response to cholesterol lowering therapy between men and women without cardiovascular disease is difficult to visualise. Atherosclerotic lesions in men and women are indistinguishable pathologically. ${ }^{10}$ Although women typically develop atherosclerosis more slowly than men, a subgroup of women can be identified that carry the same risk as men. ${ }^{1}$ Most of these women have multiple cardiovascular risk factors; there is no reliable evidence that they do not benefit from cholesterol lowering.

\section{Level of risk}

The current model for preventing cardiovascular disease holds that intensity of therapy should be proportional to absolute risk. ${ }^{12}$ Higher risk patients deserve the most intensive treatment because they experience the greatest reduction in absolute risk in the shortest time and most cost effectively. There is general agreement that both men
Distribution of 10 year risks for coronary heart disease from the Framingham offspring study according to age ${ }^{\star 13}$

\begin{tabular}{|c|c|c|c|c|c|}
\hline \multirow{2}{*}{$\begin{array}{l}10 \text { year risk } \\
\text { of heart disease } \\
\text { (\%) }\end{array}$} & \multicolumn{5}{|c|}{ Age (years) } \\
\hline & $30-39$ & $40-49$ & $50-59$ & $60-69$ & $70-79$ \\
\hline \multicolumn{6}{|l|}{ Men } \\
\hline$<10$ & 98 & 87 & 63 & 29 & 12 \\
\hline $10-20$ & 2 & 11 & 30 & 43 & 39 \\
\hline 220 & 0 & 2 & 7 & 28 & 49 \\
\hline \multicolumn{6}{|l|}{ Women } \\
\hline$<10$ & 100 & 100 & 94 & 87 & 78 \\
\hline $10-20$ & 0 & 0 & 5 & 10 & 17 \\
\hline 120 & 0 & 0 & 1 & 3 & 5 \\
\hline
\end{tabular}

and women with established cardiovascular disease are at high risk and should get intensive cholesterol lowering therapy. ${ }^{11}{ }^{12}$ The US National Cholesterol Education Program guidelines define high risk as a 10 year risk of coronary heart disease greater than $20 \% .{ }^{12}$ The essential issue here is whether women as well as men should be considered for cholesterol lowering drugs when their 10 year risk is $10-20 \%$-that is, moderately high. These people will not have established coronary heart disease, and treatment constitutes primary prevention.

\section{To maximise cholesterol lowering, combining dietary therapy with drug treatment is prudent}

Let us consider the magnitude of the issue. Data from the Framingham Offspring Study show that only a few women without cardiovascular disease have a 10 year risk of coronary heart disease over $10 \%$, and most of them are over 60 (table)..$^{13}$ Many will be smokers or have diabetes or multiple risk factors. Estimating absolute risk before starting drug therapy will ensure that lower risk women will not be treated inappropriately with drugs.

\section{Artifical distinctions}

Most investigators believe that primary and secondary prevention is an artificial distinction that should give way to a strategy based on absolute risk for future cardiovascular events, regardless of whether previ- ous events have occurred. Clinical trials that have included both men and women at moderately high risk have shown overall risk reduction from cholesterol lowering therapy. ${ }^{14-16}$ But post-hoc analyses limited to women failed to show significant risk reduction because of a lack of statistical power. Not enough women were included to provide a definite result. Some investigators take this result to mean lack of efficacy. ${ }^{8}$ But without adequate power, the results simply are not informative. The relatively small numbers of women who are at moderately high risk and who are available for clinical trials make it unlikely that a definitive answer will be obtained anytime soon (table). Other approaches to decision making are therefore required.

\section{Acting on evidence}

Until a large-scale clinical trial is carried out to test the efficacy of cholesterol lowering in women at moderately high risk we have two options: to exclude such women from drug treatment, or to consider treatment based on a combination of clinical trial evidence and epidemiological data. Since a substantial proportion of women ultimately develop cardiovascular disease and die from it, withholding treatment in moderately high risk women that has proved protective in men at similar risk and in women at high risk seems to be stretching the restrictions of "evidence based medicine" beyond reason.

The argument that women in the moderately high risk category should be treated with dietary therapy alone is no solution. We have even less clinical trial evidence for benefit of diet treatment in this group than for drug treatment. Of course, to maximise cholesterol lowering, combining dietary therapy with drug treatment is prudent.

In summary, cholesterol lowering drugs should be avoided in most lower risk women. But in those who have multiple cardiovascular risk factors and who are projected to be at moderately high risk, use of drugs such as statins should not be ruled out.

Competing interests: SMG has been a consultant to the following companies that market statins: Merck, Pfizer, Bristol Myers Squibb, and Astra Zeneca.

References are in the full version on bmj.com 